\title{
Multimode Equivalent Networks for Shielded Microwave Circuits With Thick Metallizations
}

\author{
Celia Gomez Molina ${ }^{\circledR}$, Fernando Quesada Pereira ${ }^{\circledR}$, Member, IEEE, Stephan Marini ${ }^{\circledR}$, Senior Member, IEEE, \\ Alejandro Alvarez Melcon ${ }^{\circledR}$, Senior Member, IEEE, Vicente E. Boria ${ }^{\circledR}$, Fellow, IEEE, \\ and Marco Guglielmi ${ }^{\circledR}$, Life Fellow, IEEE
}

\begin{abstract}
In this article, we show how the multimode equivalent network (MEN) technique can be extended to the analysis of multilayer boxed planar microwave circuits that are built using a thick metallization. This analysis is carried out considering the thick metallic circuit as a length of the uniform waveguide. The problem is then divided into two waveguide steps (or junctions). We first obtain the equivalent network of the two junctions. Then, we cascade them to obtain the MEN of the complete circuit, thereby including the effect of finite thickness rigorously. A key aspect of the formulation that we propose is that it includes ports in the transverse plane of the circuit. In addition to theory, several shielded microwave filters using a thick metallization are also analyzed. An in-depth study of the numerical convergence is also performed for two practical examples. Simulation results are shown to be in good agreement with both commercial simulation tools and measurements, thereby fully validating our theoretical formulation.
\end{abstract}

Index Terms - Integral equations, method of moments (MoM), microwave filters, multimode equivalent network (MEN), numerical methods, thick planar microwave circuits, transversal filters.

\section{INTRODUCTION}

$\mathbf{I}$ $\mathrm{N}$ THE microwave industry, numerical techniques are of great interest since they allow fast and accurate analysis of the structures under design. Several numerical techniques are indeed described in the technical literature [1], [2] and are also implemented in commercial tools, such as ANSYS HFSS, CST Studio Suite, or FEST3D. Depending on the structure

Manuscript received April 9, 2020; revised June 24, 2020; accepted July 25, 2020. This work was supported in part by the Ministerio de Educación, Cultura y Deporte, Spanish Government, under Grant FPU15/02883; and in part by the Ministerio de Ciencia e Innovación through the sub-projects C41, C42 and C43 of the coordinated project under Grant PID2019-103982RB. (Corresponding author: Celia Gomez Molina.)

Celia Gomez Molina, Fernando Quesada Pereira, and Alejandro Alvarez Melcon are with the Department of Information Technologies and Communications, Universidad Politécnica de Cartagena, 30202 Cartagena, Spain (e-mail: celia.gomez@upct.es; fernando.quesada@upct.es; alejandro.alvarez@upct.es ).

Stephan Marini is with the Department of Physics, Systems Engineering and Signal Theory, Universidad de Alicante, 03690 Alicante, Spain (e-mail: smarini@ua.es).

Vicente E. Boria and Marco Guglielmi are with the Research Development Institute on Telecommunication and Multimedia Applications, Universidad Politécnica de Valencia, 46022 Valencia, Spain (e-mail: vboria@dcom.upv.es; marco.guglielmi@iteam.upv.es).

Color versions of one or more of the figures in this article are available online at http://ieeexplore.ieee.org.

Digital Object Identifier 10.1109/TMTT.2020.3016396 under analysis, one tool may be more suitable than another. For example, general numerical techniques, such as the finite element method [3] and finite-difference time domain [4], are able to analyze complex geometries at the expense, most of the time, of consuming significant computational resources. Others, such as those based on modal methods and integral equations (IEs) [5], are usually very efficient but can only cope with a more restricted class of geometries.

Within this last family of numerical methods, we can find the multimode equivalent network (MEN) technique [6]. This technique is based on the analysis of each discontinuity present in the structure through a rigorous multimode equivalent circuit. The formulation starts by imposing the boundary conditions for the electromagnetic (EM) fields to obtain the integral equation that represents the problem. Once the IE is solved through the well-known method of moments and Galerkin procedure (MoM-Galerkin), we obtain a generalized impedance (or admittance) coupling matrix that represents the interaction between the modes excited on both sides of each discontinuity. To analyze the complete structure, the individual equivalent networks are then cascaded to form a final global network.

It is interesting to note that the MEN technique starts with the expansion of the EM fields as a summation of modes, in the same way as the mode-matching (MM) technique [1]. However, in the MM technique, the global boundary conditions are then applied, and the solution of the expansion coefficients is obtained directly through the solution of the global system representing the complete device. In the MEN technique, on the contrary, we first formulate and solve an IE using the auxiliary magnetic current densities in the aperture. We then relate the EM fields with the modal voltages and currents, and we interpret the resulting relations with a MEN. The individual networks are then cascaded to obtain the performance of the complete device. As a result, we can verify the convergence of each separate MEN and avoid completely any relative convergence problem.

The MEN technique has been traditionally applied to waveguide devices [6], [7], obtaining very efficient and accurate results. Additional work has also been done to improve the computational performance of MEN representations [8], [9]. Waveguide components are normally composed of cascaded 
uniform waveguide sections (for instance, cavities and coupling windows). The various waveguide junctions are then analyzed separately to derive the relevant MENs. In addition, in [10], metal gratings inside waveguides are analyzed using a zero-thickness formulation. A common feature of the formulations discussed so far is that the exciting power is carried by modes propagating in the input and output (I/O) waveguide sections. This is indeed appropriate for the analysis of waveguide components. However, planar microwave circuits are today very popular for a large number of applications. In this context, therefore, research was recently carried out to extend the MEN formulation to the analysis of multilayer boxed planar devices [11], [12] and to include also lateral excitation ports that could model the excitation of planar circuits through real-coaxial connectors [13], [14]. However, the formulation presented in [11], [12] is restricted to zero-thickness metallic patches with a rectangular shape. Gómez Molina et al. [15] extended the analysis to multilayer planar devices that consist of arbitrarily shaped zerothickness metallizations. This is possible thanks to the combination of the MEN formulation and the BI-RME method [16], similar to what has already been done for waveguide problems [17].

In many cases of practical interest, however, the thickness of the metallization cannot be neglected, and the zero-thickness formulation described in [15] is no longer accurate. If the thickness is nonnegligible but is electrically small, alternative IE techniques, like the one described in [18], can be used to transform the thin aperture or metallization into an equivalent zero-thickness object. However, if the metallization is electrically thick, a more accurate formulation is required. This is indeed the case, for instance, when planar circuits are analyzed at very high frequencies. In this situation, the thickness becomes electrically important and, therefore, it must be included explicitly in the computations in order to obtain accurate results. Furthermore, a new class of thick bar resonators has been recently proposed to implement wideband filters [19]. The thicker metallic resonators exhibit better unloaded quality factors than regularly printed microstrip resonators. Moreover, higher coupling levels (between resonators and with the I/O ports) than the ones obtained with thinner (standard) planar microstrip technology can be achieved. However, since the thickness of the resonators is electrically large even at microwave frequencies, the zero-thickness formulation described in [15] is not suitable anymore. In this context, therefore, the objective of this article is to extend the stateof-the-art of the analysis of planar microwave circuits by discussing a MEN formulation that can analyze rigorously planar microwave circuits based on thick metallizations, including also coaxial I/O ports, and several dielectric layers, as shown in Fig. 1. The effect of losses due to metal and dielectric materials of the multilayer circuits will also be accounted for.

This article is organized as follows. In Section II, we first describe the analysis of thick discontinuities, including ports in the transverse plane. We then explain how to cascade the equivalent networks to build the final model that describes the complete structure. In Section III, we study the numerical convergence of the formulation for two in-line microwave

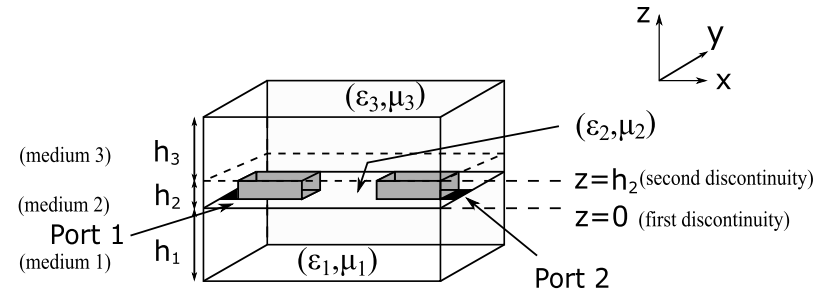

Fig. 1. Three-dimensional view of the structure under analysis. The metallization has a nonnegligible thickness $\left(h_{2}\right)$. The structure is excited by two lateral ports (Ports 1 and 2), at the interface $z=0$.

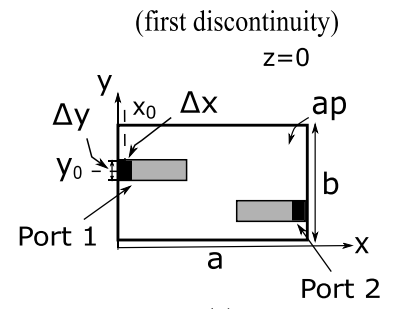

(a)

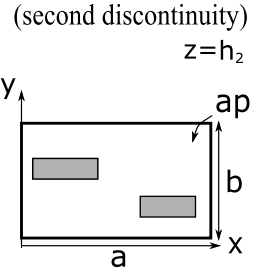

(b)
Fig. 2. Top view of the two discontinuities present in the structure shown in Fig. 1. (a) First discontinuity (at $z=0$ ) contains the lateral excitation ports (dark areas) that excite the structure. (b) Second discontinuity (at $z=h_{2}$ ) only contains the metallizations (gray areas).

filters implemented with thick coupled lines. In Section IV, we compare the simulation results for a transversal fourpole filter with measurements obtained from a manufactured prototype, showing very good agreement, and thereby fully validating the new MEN formulation. Finally, a summary of the results obtained is provided in the conclusions.

\section{MEN THEORY}

The goal of this section is to extend the MEN technique to the analysis of devices containing transverse ports and a combination of several thick metallizations and dielectric layers, as shown in Fig. 1. Since the metallizations have a nonnegligible thickness ( $h_{2}$ in Fig. 1$)$, the zero-thickness MEN formulation described in [15] cannot be used to analyze such structures. As we can see in Fig. 1, the structure can be decomposed into two discontinuities (one at $z=0$ and the other one at $z=h_{2}$ ).

A top view of the two discontinuities is shown in Fig. 2. The first discontinuity [see Fig. 2(a)] is analyzed using the MEN formulation with transverse ports, as described in Section II-A. It should be emphasized that the transverse ports can be either external or internal, as discussed in [15]. In Fig. 2(a), we show two external ports to model the coaxial I/O connectors used to excite real-microwave components [13], [14]. However, the treatment of internal ports, which can be used to connect the metallization to external lumped-element components, is identical. The second discontinuity present in the structure of Fig. 1 [see Fig. 2(b)] is analyzed using the traditional MEN for waveguide junctions [6].

It is important to note that this is only an example of a possible microwave planar circuit with thick metallizations. The formulation presented in this work is not restricted to 
any particular shape. In fact, the shape can be arbitrary thanks to the use of the BI-RME method [17]. Once both MENs are obtained, they are cascaded, as explained in Section II-B, to compute the final network that characterizes the complete structure in Fig. 1.

\section{A. MEN for Discontinuities of Thick Junctions Including Transverse Ports}

In this section, we derive the general MEN formulation for 2-D discontinuities of thick junctions that include transverse ports [as shown in Fig. 2(a)]. The formulation starts by applying the boundary conditions on the tangential components of the magnetic field $\mathbf{H}_{t}^{(\delta)}$ in the aperture at $z=0$, where $\delta$ represents the regions on both sides of the discontinuity $(\delta=1$ or $\delta=2$ for $z<0$ or $z>0$, respectively). In this condition, we include the lateral excitation through the surface electric current density $\mathbf{J}_{\text {exc }}$ as follows [15]:

$$
\mathbf{H}_{t}^{(1)}(s)-\mathbf{H}_{t}^{(2)}(s)=\mathbf{z}_{0} \times \mathbf{J}_{\mathrm{exc}}(s) .
$$

In (1), $s$ indicates a point in the waveguide cross section of the discontinuity, and $\mathbf{z}_{0}$ is the unit vector in the direction of the $z$-axis (see Fig. 1).

For simplicity, only one transverse port is considered explicitly in this development, but the extension to more than one port (either external or internal) is straightforward. To continue, we use the well-known modal expansion formalism in [6] to expand the magnetic field, and the pulse model in [20] for the surface electric current density representing the excitation [see Fig. 2(a)]. We also separate the accessible modes from the localized ones, as indicated in [6], to obtain

$$
\begin{aligned}
\sum_{n=1}^{N(1)} I_{n}^{(1)} \mathbf{h}_{n}^{(1)}(s)-\sum_{n=1}^{N(2)} I_{n}^{(2)} \mathbf{h}_{n}^{(2)}(s) & \\
& +\frac{I_{0}}{\Delta y} \prod\left(\frac{x-x_{0}}{\Delta x}\right)\left(\frac{y-y_{0}}{\Delta y}\right) \mathbf{y}_{0} \\
= & \sum_{m=N(1)+1}^{+\infty} Y_{m}^{(1)} V_{m}^{(1)} \mathbf{h}_{m}^{(1)}(s)+\sum_{m=N(2)+1}^{+\infty} Y_{m}^{(2)} V_{m}^{(2)} \mathbf{h}_{m}^{(2)}(s)
\end{aligned}
$$

where $\mathbf{h}_{m}^{(\delta)}(s)$ is the magnetic vector modal function of mode $m$ in the medium $(\delta)$, and $I_{m}^{(\delta)}$ represents the total modal current. In (2), $V_{m}^{(\delta)}$ and $Y_{m}^{(\delta)}$ represent the total modal voltage and the characteristic modal admittance, respectively. In addition, $N(\delta)$ is the number of accessible modes considered in each region [9]. Regarding the pulse, $\left(x_{0}, y_{0}\right)$ denotes the position of the center of the pulse, and $\Delta x$ and $\Delta y$ are the widths of the pulse in the $x$-axis and $y$-axis, respectively [see Fig. 2(a)].

It is now important to note a difference between the zerothickness formulation presented in [15] and the formulation discussed in this article. The difference is that the modal voltages on both sides of the discontinuity $V_{m}^{(1)}$ and $V_{m}^{(2)}$ are not equal. In addition, the magnetic vector modal functions in medium 1, i.e., $\mathbf{h}_{m}^{(1)}(s)$, are those corresponding to the rectangular waveguide, while, in medium 2 , the functions $\mathbf{h}_{m}^{(2)}(s)$ are the modes of the aperture (with the arbitrary shape) that are obtained using the BI-RME method.

To continue the formulation, we now use the relation between the modal voltage $V_{m}^{(\delta)}$ and the unknown electric field in the aperture $\mathbf{E}$

$$
V_{m}^{(\delta)}=\int_{\text {ap }}\left[\mathbf{z}_{0} \times \mathbf{E}\left(s^{\prime}\right)\right] \cdot \mathbf{h}_{m}^{(\delta) *}\left(s^{\prime}\right) d s^{\prime}
$$

where $a p$ is the aperture at the junction (see Fig. 2). Due to the linearity of the problem, the electric field $\mathbf{E}$ must be proportional to the excitation, so that we can write

$$
\begin{aligned}
{\left[\mathbf{z}_{0} \times \mathbf{E}\left(s^{\prime}\right)\right] } & \\
& =\sum_{n=1}^{N(1)} I_{n}^{(1)} \mathbf{M}_{n}^{(1)}\left(s^{\prime}\right)-\sum_{n=1}^{N(2)} I_{n}^{(2)} \mathbf{M}_{n}^{(2)}\left(s^{\prime}\right)+I_{0} \mathbf{M}_{0}\left(s^{\prime}\right)
\end{aligned}
$$

where $\mathbf{M}_{n}^{(\gamma)}$ and $\mathbf{M}_{0}$ are the unknowns of our problem, and $\gamma$ represents the regions on both sides of the discontinuity from the source point of view $(\gamma=1$ or $\gamma=2$ for $z<0$ or $z>0$, respectively). Substituting now (4) into (3), and using the resulting equation in (2), we obtain the system of IEs that represents the problem under study

$$
\begin{gathered}
\mathbf{h}_{n}^{(\gamma)}(s)=\int_{a p} \mathbf{M}_{n}^{(\gamma)}\left(s^{\prime}\right) \cdot \overline{\mathbf{K}}\left(s, s^{\prime}\right) d s^{\prime} \\
\frac{1}{\Delta y} \prod\left(\frac{x-x_{0}}{\Delta x}\right)\left(\frac{y-y_{0}}{\Delta y}\right) \mathbf{y}_{0}=\int_{a p} \mathbf{M}_{0}\left(s^{\prime}\right) \cdot \overline{\mathbf{K}}\left(s, s^{\prime}\right) d s^{\prime}
\end{gathered}
$$

where $\overline{\mathbf{K}}\left(s, s^{\prime}\right)$ is the kernel of the two previous IEs

$$
\overline{\mathbf{K}}\left(s, s^{\prime}\right)=\sum_{\delta=1}^{2} \sum_{m=N(\delta)+1}^{+\infty} Y_{m}^{(\delta)} \mathbf{h}_{m}^{(\delta) *}\left(s^{\prime}\right) \mathbf{h}_{m}^{(\delta)}(s) .
$$

To increase the computational efficiency, the kernel is evaluated using Kummer's technique, as described in [8]. Thanks to this transformation, the kernel in (7) is split into a dynamic and a static part. As a result, the dynamic part, that needs to be recomputed for each frequency point, exhibits a much faster convergence rate. Furthermore, the important computational burden in the static part of the kernel is independent of frequency, and it just needs to be computed only once for all the analysis frequency range.

Finally, we write the modal voltages in terms of the modal currents as

$$
\begin{gathered}
V_{0}=Z_{0,0} I_{0}+\sum_{n=1}^{N(1)} I_{n}^{(1)} Z_{0, n}^{(1)}+\sum_{n=1}^{N(2)} I_{n}^{(2)} Z_{0, n}^{(2)} \\
V_{m}^{(\delta)}=Z_{m, 0}^{(\delta)} I_{0}+\sum_{n=1}^{N(1)} I_{n}^{(1)} Z_{m, n}^{(\delta, 1)}+\sum_{n=1}^{N(2)} I_{n}^{(2)} Z_{m, n}^{(\delta, 2)}
\end{gathered}
$$

where $Z_{0,0}, Z_{0, n}^{(\gamma)}, Z_{m, 0}^{(\delta)}$, and $Z_{m, n}^{(\delta, \gamma)}$ are the elements of the multimode impedance coupling matrix for the discontinuity under study.

As shown in (6), the inclusion of the transverse port as the excitation of the structure leads to a new IE, where the excitation is the pulse function introduced in (1). This is an important 


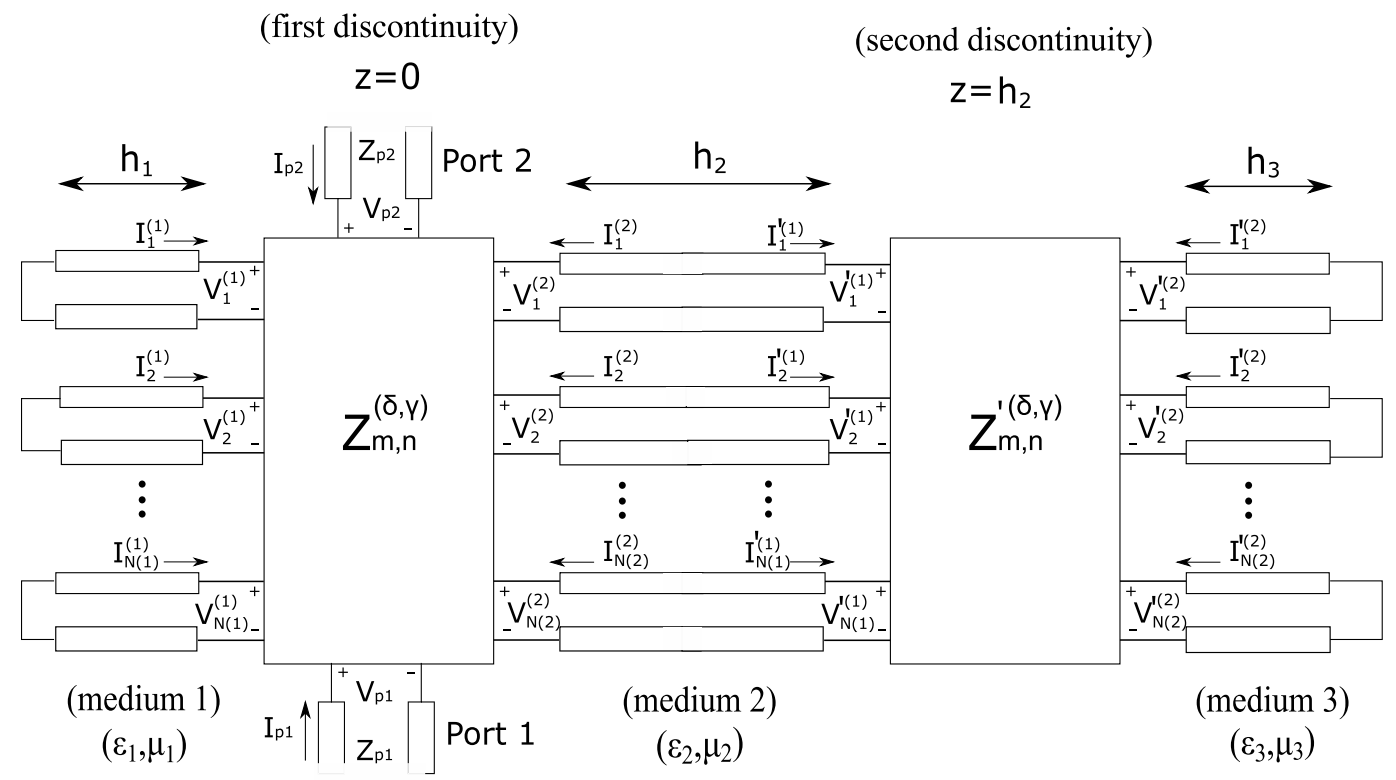

Fig. 3. Final equivalent network representation for the structure shown in Fig. 1, using the MEN formalism. The discontinuities are characterized by their MEN coupling matrices. The modes are characterized by transmission lines in different regions. Modes in media 1 and 3 are terminated with a short-circuit to model the top and bottom metal covers of the box enclosure (see Fig. 1). The coaxial excitations are represented with transmission lines of characteristic impedances $Z_{p 1}$ and $Z_{p 2}$ for Ports 1 and 2, respectively.

difference with respect to the traditional waveguide junctions normally used in the analysis of waveguide components [6]. Note that the presence of the new excitation term results into the new elements $\left(Z_{0,0}, Z_{0, n}^{(\gamma)}\right.$ and $\left.Z_{m, 0}^{(\delta)}\right)$ in the coupling matrix that take into account the interactions between the modes on both sides of the discontinuity and the new lateral excitation port.

Finally, the MoM-Galerkin procedure can be used to solve the system of IEs in (5) and (6), as reported in [6] and [15]. In this case, we use the magnetic vector modal functions of modes in medium 2 (see Fig. 1) as basis and test functions. The required modes are computed very efficiently using the BI-RME method [16], [17].

\section{B. Final MEN Assembly}

Once we have computed the equivalent network for both discontinuities in Fig. 2, we can cascade them, as shown in Fig. 3.

The first discontinuity contains, in addition, two ports that represent the lateral excitations of the structure [Ports 1 and 2 in Fig. 1, represented with dark areas in Fig. 2(a)]. The modes in the different media are represented by transmission lines (where the propagation constant and the characteristic impedance are those corresponding to the mode in that medium). The lengths of the transmissions lines correspond to the heights $\left(h_{1}, h_{2}\right.$ and $\left.h_{3}\right)$ in Fig. 1. As we can see in Fig. 3, the modes that represent the first and the last layer $\left(h_{1}\right.$ and $\left.h_{3}\right)$ are short-circuited to represent the top and bottom covers of the external metal housing. To analyze the complete structure using this final network, we write the external voltages $\left(V_{n}^{(1)}\right.$ and $\left.V_{n}^{\prime(2)}\right)$ and the port voltages $\left(V_{p 1}\right.$ and $\left.V_{p 2}\right)$ in terms of the external current $\left(I_{n}^{(1)}\right.$ and $\left.I_{n}^{\prime(2)}\right)$ and the port currents ( $I_{p 1}$ and $\left.I_{p 2}\right)$, obtaining the following system of linear equations:

$$
\left[\begin{array}{c}
V_{p 1} \\
0 \\
0 \\
\cdot \\
\cdot \\
0
\end{array}\right]=\left[\begin{array}{ccccc}
Z_{p 1, p 1}^{T} & Z_{p 1, p 2}^{T} & Z_{p 1,1}^{T} & \cdot & Z_{p 1, N}^{T} \\
Z_{p 2, p 1}^{T} & Z_{p 2, p 2}^{T} & Z_{p 2,1}^{T} & \cdot & Z_{p 2, N}^{T} \\
Z_{1, p 1}^{T} & Z_{1, p 2}^{T} & Z_{1,1}^{T} & \cdot & Z_{1, N}^{T} \\
\cdot & \cdot & & \cdot & \cdot \\
Z_{N, p 1}^{T} & Z_{N, p 2}^{T} & Z_{N, 1}^{T} & \cdot & Z_{N, N}^{T}
\end{array}\right]\left[\begin{array}{c}
I_{p 1} \\
I_{p 2} \\
I_{1}^{(1)} \\
\cdot \\
\cdot \\
I_{N(2)}^{\prime(2)}
\end{array}\right]
$$

where we have loaded the modes with the corresponding total impedance, and Port 2 is loaded with the appropriate reference impedance (in our case $Z_{p 2}$ ). We then excite through Port 1 and solve the linear system to obtain the input admittance $\left(Y_{\text {in }}=I_{p 1} / V_{p 1}\right)$ and the transadmittance between ports $\left(Y_{\text {tran }}=I_{p 2} / V_{p 1}\right)$. The S-parameters are then calculated in terms of $Y_{\text {in }}$ and $Y_{\text {tran }}$, as shown in the following:

$$
\begin{aligned}
S_{11} & =\frac{1-Z_{p 1} Y_{\text {in }}}{1+Z_{p 1} Y_{\text {in }}} \\
S_{21} & =Y_{\operatorname{tran}} \frac{\left(-2 Z_{p 2}\right)}{1+Z_{p 1} Y_{\text {in }}} \sqrt{\frac{Z_{p 1}}{Z_{p 2}}} .
\end{aligned}
$$

\section{NumericAl CONVERGEnCE Study}

In this section, we analyze two filters that are based on the basic geometry shown in Fig. 1. The filters are described in detail in [19]. A general schematic of the structure under analysis is shown in Fig. 4.

The filters use three thick metallic bars as resonators. The first and the third bars are coupled by proximity to the second bar. Due to the nonnegligible thickness of the resonators $\left(h_{2}\right)$, a strong capacitive coupling is induced between bars, 


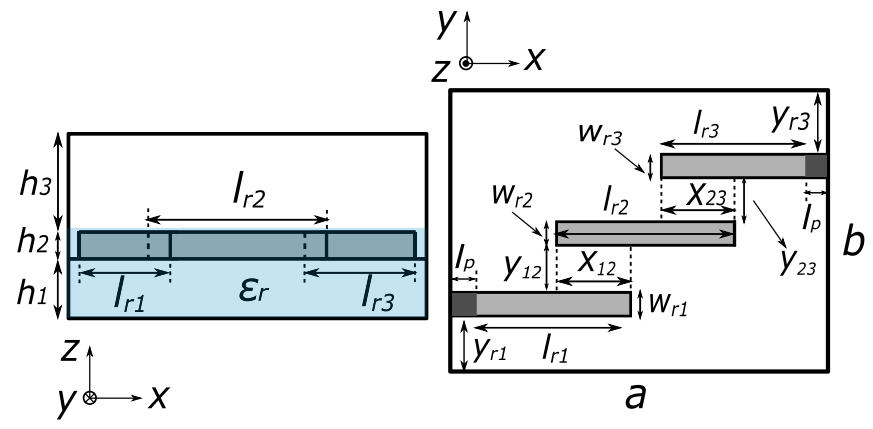

Fig. 4. Side (left) and top (right) views of the filters under study. Dark areas $\left(l_{p}\right)$ represent lumped ports that model the direct connection of the coaxial pins to the first and last resonators (Ports 1 and 2). In this case, $\Delta x=l_{p}$ and $\Delta y=W_{r 1}$.

TABLE I

Physical Dimensions of THE Two In-Line Filters STUdied In This Section, AS Defined IN Fig. 4

\begin{tabular}{|c|c|c|}
\hline $\begin{array}{c}\text { Parameters } \\
\text { of the filter }\end{array}$ & $\begin{array}{c}\text { Dimensions } \\
\text { first filter } \\
\text { (in-line) }\end{array}$ & $\begin{array}{c}\text { Dimensions } \\
\text { second filter } \\
\text { (in-line) }\end{array}$ \\
\hline \hline$h_{1}=h_{3}$ & $10 \mathrm{~mm}$ & $10 \mathrm{~mm}$ \\
$h_{2}$ & $2 \mathrm{~mm}$ & $0.5 \mathrm{~mm}$ \\
$l_{r 1}=l_{r 3}$ & $16.6 \mathrm{~mm}$ & $15.25 \mathrm{~mm}$ \\
$l_{r 2}$ & $30.65 \mathrm{~mm}$ & $29.3 \mathrm{~mm}$ \\
$w_{r 1}=w_{r 2}=w_{r 3}$ & $2 \mathrm{~mm}$ & $2 \mathrm{~mm}$ \\
$y_{r 1}=y_{r 3}$ & $16.8 \mathrm{~mm}$ & $16.8 \mathrm{~mm}$ \\
$y_{12}=y_{23}$ & $4.5 \mathrm{~mm}$ & $4.5 \mathrm{~mm}$ \\
$x_{12}=x_{23}$ & $11.325 \mathrm{~mm}$ & $10.7 \mathrm{~mm}$ \\
$a$ & $42.2 \mathrm{~mm}$ & $39.4 \mathrm{~mm}$ \\
$b$ & $48.6 \mathrm{~mm}$ & $48.6 \mathrm{~mm}$ \\
$\varepsilon_{r}$ & 1.05 & 1.05 \\
$Z_{p 1}=Z_{p 2}$ & $45 \Omega$ & $40 \Omega$ \\
\hline \multicolumn{2}{|c}{} \\
\hline
\end{tabular}

obtaining higher interresonator couplings in comparison to standard microstrip filters. In [19], it is also demonstrated that the unloaded quality factor of these thick resonators is substantially higher as compared with regularly printed microstrip resonators. Moreover, the I/O couplings are implemented through the direct connection of the lateral ports (that model the coaxial connectors) to the first and last thick bars. Following this approach, the traditional I/O feeding lines are not required. A foam substrate with $\left(\varepsilon_{r}=1.05\right)$ is used to avoid the excitation of box modes. The resonators of the filters are, thus, formed by the three thick bars. The result is two third-order in-line filters, whose dimensions are collected in Table I.

The numerical behavior of the MEN technique for standard waveguide problems has been extensively studied in other publications, for instance, in [9]. For this new problem, the computational novelty is the study of the number of accessible modes that are required as a function of the thickness $h_{2}$ (medium 2, see Fig. 3) for accurate characterization of the whole circuit. As shown in Fig. 3, the number of accessible modes determines the size of the equivalent network. It is important to remember that the higher order modes (localized modes) that have been loaded with their corresponding modal admittances are taken into account rigorously in (7) by using Kummer's transformation [8].

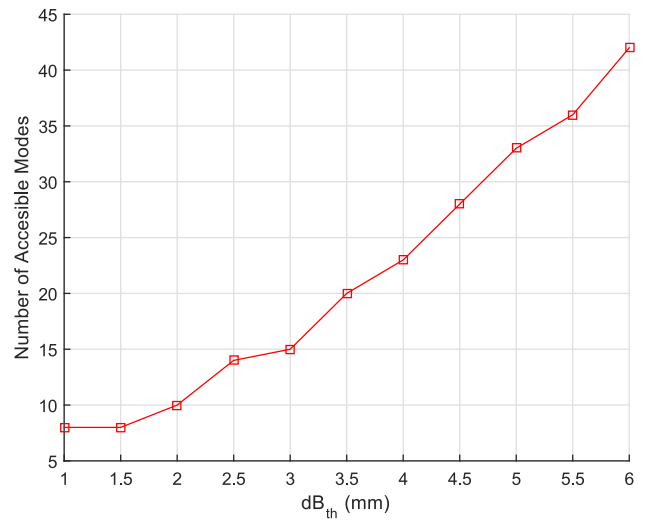

Fig. 5. Number of accessible modes that are necessary according to the specified threshold $\left(\mathrm{dB}_{\text {th }}\right)$. This test is carried out for an operating frequency of $f_{0}=5 \mathrm{GHz}$ and thickness $h_{2}$ equal to $2 \mathrm{~mm}$.

To show the behavior of this MEN formulation, we now perform two numerical studies. In both of them, we use the theory described in [9] that allows selecting the right number of accessible modes $[N(1)=N(2)=N(3)=N]$ according to a power attenuation threshold in $\mathrm{dB}$ (called $\left.\mathrm{dB}_{\mathrm{th}}\right)$. Using this strategy, the number of accessible modes in each region will be composed of all propagating modes plus the evanescent modes that undergo a total attenuation level, when reaching the next discontinuity, that is smaller than the specified threshold $\mathrm{dB}_{\text {th }}$.

\section{A. Number of Accessible Modes According to $d B_{t h}$}

In the first test, we study the numerical convergence of this formulation according to the parameter $\mathrm{dB}_{\text {th }}$, for a fixed value of the metallization thickness. For this test, we use the first in-line filter shown in Table I. In this case, the metallizations that compose the filter have a thickness of $h_{2}$ equal to $2 \mathrm{~mm}$.

Based on the mechanism proposed in [9], we show in Fig. 5 the required number of accessible modes as a function of the specified threshold $\mathrm{dB}_{\text {th }}$. As shown in Fig. 5, when the parameter $\mathrm{dB}_{\text {th }}$ is larger, more evanescent modes need to be considered as accessible ones. This will increase the size of the total matrix in (10), thus increasing the computational effort. This is confirmed in Table II that specifies the number of accessible modes required to reach different levels of threshold and the related computational time for each case. To check how the value of the threshold affects the numerical accuracy of the technique, we plot in Fig. 6 the filter response for different values of $\mathrm{dB}_{\text {th }}$.

As we can see, the results are stable with values of the threshold $\mathrm{dB}_{\text {th }}>4 \mathrm{~dB}$. Selecting $\mathrm{dB}_{\text {th }}=4 \mathrm{~dB}$ requires $N=$ 23 accessible modes. The S-parameters simulated using the MEN formulation are compared with the results obtained with HFSS in Fig. 7. As we can see, the two software tools provide similar results. This validates, at the same time, both the theory and the threshold value we have selected.

In Fig. 7, we also show the simulation results for this filter using the zero-thickness MEN formulation discussed in [15]. As we can see, the zero-thickness results are clearly different from the responses obtained using both HFSS and the proposed thick MEN formulation. This is because the thickness of 
TABLE II

Number of AcCessible Modes And Computation Time ACCORDING TO THE SELECTED THRESHOLD $\mathrm{dB}_{\text {th }}$

\begin{tabular}{|c|c|c|}
\hline $\begin{array}{c}\text { Attenuation Threshold } \\
\left.\mathrm{dB}_{t h} \text { (in } \mathrm{dB}\right)\end{array}$ & $\begin{array}{c}\text { Number of accessible modes } \\
(N)\end{array}$ & $\begin{array}{c}\text { Time } \\
\text { (in seconds) }\end{array}$ \\
\hline \hline 1 & 8 & 6.84 \\
1.5 & 8 & 6.84 \\
2 & 10 & 7.88 \\
2.5 & 14 & 10.15 \\
3 & 15 & 10.26 \\
3.5 & 20 & 13.86 \\
4 & 23 & 14.52 \\
4.5 & 28 & 17.09 \\
5 & 33 & 20.57 \\
5.5 & 36 & 22.38 \\
6 & 42 & 26.64 \\
\hline
\end{tabular}

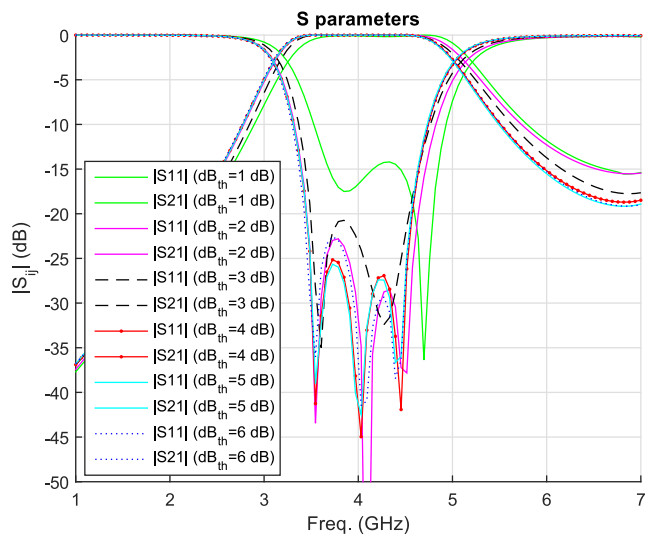

Fig. 6. S-parameter response of the first in-line filter (with $h_{2}=2 \mathrm{~mm}$ ) for different values of the threshold $\mathrm{dB}_{\text {th }}$.

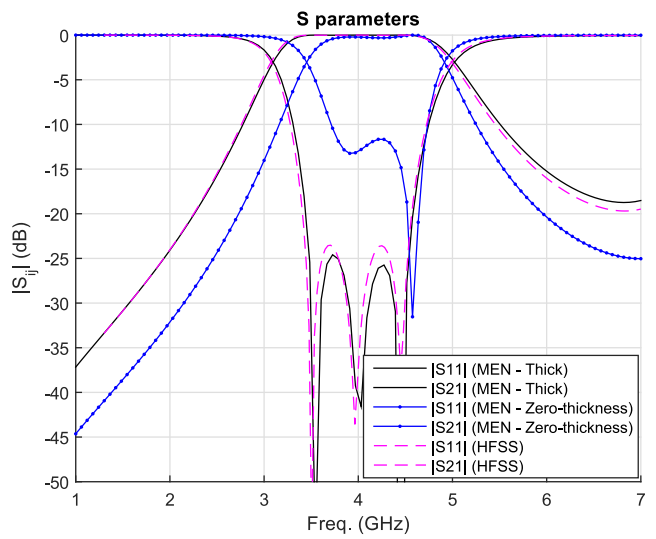

Fig. 7. Magnitude of the S-parameters for the first in-line filter (with $h_{2}=2$ $\mathrm{mm}$ ) computed using the thick MEN formulation as compared with the results from HFSS. The simulation results using the zero-thickness MEN formulation described in [15] are also included for comparison.

the metallizations $\left(h_{2}=2 \mathrm{~mm}\right)$ is electrically large and cannot be neglected. This result confirms that for this filter, zerothickness approximations are not accurate. Therefore, it also demonstrates the importance of the formulation proposed in this article to analyze this kind of microwave components.

\section{B. Number of Accessible Modes According to the Thickness $h_{2}$}

The second test is related to the number of accessible modes required according to the thickness of the metallizations

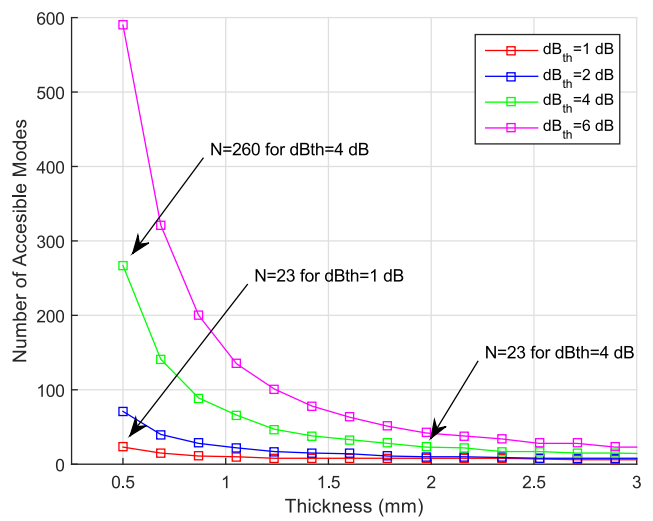

Fig. 8. Necessary number of accessible modes according to the thickness of the metallization $\left(h_{2}\right.$, see Fig. 4). This test is done for different power thresholds and for an operating frequency equal to $f_{0}=5 \mathrm{GHz}$.

$\left(h_{2}\right.$, medium 2 in Fig. 1) for a fixed value of the power threshold $\mathrm{dB}_{\text {th. }}$. In Fig. 8, we show the required number of accessible modes according to this thickness $\left(h_{2}\right)$, and for different values of the power threshold.

As we can see in Fig. 8, more accessible modes are required when the metallization is thinner. According to this test, a filter with metallization thickness $h_{2}=0.5 \mathrm{~mm}$ would require 260 accessible modes in the equivalent network to maintain the same power attenuation threshold, as in the previous example (i.e., $\mathrm{dB}_{\mathrm{th}}=4 \mathrm{~dB}$ ). This indicates that the proposed technique becomes less efficient for thinner metallizations. However, when the thickness decreases, the condition on the power threshold can be relaxed. To show that this is indeed the case, we have designed a second in-line filter but with a thinner metallization of $h_{2}=0.5 \mathrm{~mm}$. After optimization, the dimensions of this filter are shown in Table I (second filter). A similar numerical study for this new filter leads to a required power threshold of only $\mathrm{dB}_{\text {th }}=1 \mathrm{~dB}$ to obtain good convergence. As shown in Fig. 8, this threshold value requires only $N=23$ accessible modes. In Fig. 9 we show the S-parameters of this filter simulated using the thick MEN formulation with these numerical parameters $\left(\mathrm{dB}_{\mathrm{th}}=1 \mathrm{~dB}\right)$. The results are also compared with simulations obtained using HFSS.

From Fig. 9, we can see that the MEN results, obtained using 23 accessible modes, are in good agreement with respect to HFSS. The number of accessible modes required in this case (for $h_{2}=0.5 \mathrm{~mm}$ ) is the same as for the first in-line filter (with $h_{2}=2 \mathrm{~mm}$ ). This indicates that the efficiency of the technique does not substantially degrade if the thickness of the metallization decreases. As demonstrated, in this situation, more accessible modes are needed to maintain a certain level of the power threshold parameter $\left(\mathrm{dB}_{\mathrm{th}}=4 \mathrm{~dB}\right)$. However, this increment is compensated by the fact that the power threshold can be lowered to obtain good numerical convergence $\left(\mathrm{dB}_{\text {th }}=\right.$ $1 \mathrm{~dB}$ for $h_{2}=0.5 \mathrm{~mm}$ ).

For these two examples, we have used 180 basis and test functions in the MoM-Galerkin procedure, and 40 dynamic terms and 5000 static terms to compute the kernel of the IEs [8]. As previously mentioned, the shape of the metallization 


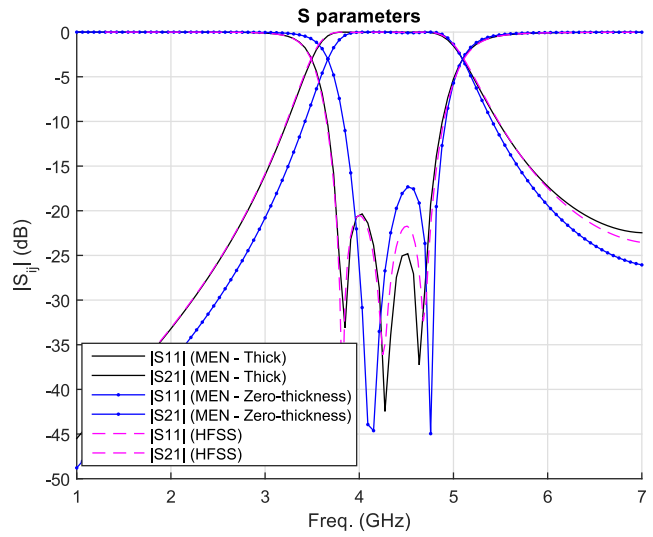

Fig. 9. Magnitude of the S-parameters for the second in-line filter (with $h_{2}=0.5 \mathrm{~mm}$ ) computed using the thick MEN formulation and compared with HFSS results. The MEN calculations are obtained with a threshold $\mathrm{dB}_{\text {th }}=$ $1 \mathrm{~dB}$. The simulation results using the zero-thickness MEN formulation described in [15] are also included for comparison.

can be arbitrary since the modes in medium 2 are computed using the BI-RME method. From the computational point of view, we first run the BI-RME module to obtain the modes of the arbitrary waveguide. We then execute the MEN code to perform the analysis of the complete structure. Using an Intel Core i7-6500U CPU @ $2.50 \mathrm{GHz}$ with $12 \mathrm{~GB}$ of RAM, the computational time required by the BI-RME code is 4 min and $46 \mathrm{~s}$, and the MEN code for 100 frequency points takes only $14.30 \mathrm{~s}$.

In Fig. 9, we have included the response obtained using the zero-thickness MEN formulation described in [15]. The zero-thickness MEN code takes $8.5 \mathrm{~s}$ for the same full-wave analysis. This significant reduction in time is due to the fact that when the zero-thickness MEN formulation is applied to single-layer microwave circuits, just one accessible mode is required in the equivalent network [15]. However, as we can see in Fig. 9, the response is not very accurate as compared with the one obtained using HFSS or the thick MEN formulation proposed in this contribution. If we compare the results in Fig. 7 for $h_{2}=2 \mathrm{~mm}$ and in Fig. 9 for $h_{2}=0.5 \mathrm{~mm}$, we can see that, as the thickness decreases, the response obtained with the zero-thickness MEN formulation is more similar to the one of the thick MEN formulation. This is confirmed in Fig. 10, where we show the responses of the same filter (second filter in Table I) but with a smaller thickness $\left(h_{2}=0.1 \mathrm{~mm}\right)$. In this case, the analysis is performed again using both the thick formulation and the zero-thickness formulation described in [15].

As we can see in Fig. 10, the resonant frequencies of the resonators and the interresonator couplings have changed with the thickness, and the filter response is not equalized anymore. However, this example shows that for thin metallizations, the response obtained using the zero-thickness formulation is similar to the thick response. When the thickness is decreased, more basis and test functions are needed to obtain accurate results, since the currents in the metallization become more singular at the edges. This, together with the required number of accessible modes $(N=23)$, results in a significant increase of the computational time for the thick formulation. On the

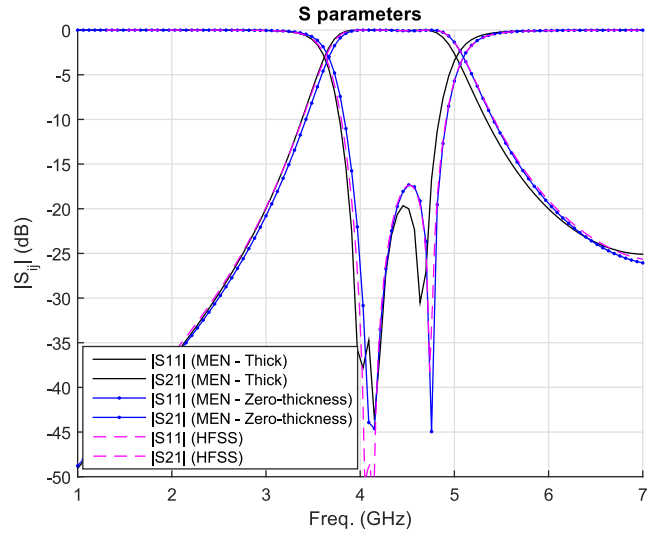

Fig. 10. Magnitude of the S-parameters for the second in-line filter but with $h_{2}=0.1 \mathrm{~mm}$, computed using the thick MEN formulation and compared to HFSS results. The MEN calculations are obtained with $N=23$. The simulation results using the zero-thickness MEN formulation described in [15] are also included for comparison.

other hand, the zero-thickness formulation just needs one accessible mode in the equivalent network, which leads to a reduction of $90 \%$ in the computational time required to achieve the same result.

From these tests, we can conclude that, when this thickness is electrically very small, the zero-thickness MEN formulation described in [15] can be used directly to analyze the structure. On the contrary, when the metallizations have a nonnegligible thickness, the MEN technique proposed in this work should be used for obtaining results with optimal accuracy. It is important to note that the formulation that we propose remains rigorous for large thicknesses, as no approximations have been introduced in the formulation of the IEs. As a consequence, the MEN remains valid even if the thickness of the metallization is increased.

\section{VALidATING EXAMPLE}

In this section, we validate through measurements the MEN theory that we propose in this contribution. For this purpose, we analyze another filter implemented using the same scheme, as the one shown in Fig. 4. However, in this case, the use of Teflon $\left(\varepsilon_{r}=2.1\right)$ as a dielectric in media 1 and 2 allows the box modes to resonate. By using the first resonance of the box, transversal coupling topologies can be implemented, as described in [19]. In order to implement a wideband response with high unloaded quality factors, the thickness of the metallic bars in this filter is $h_{2}=2 \mathrm{~mm}$. After optimization, the dimensions of the design filter are reported in Table III.

The S-parameters simulated using the MEN code and HFSS are compared with measurements (from [19]) in Fig. 11. In the MEN simulation, losses due to the dielectric in media 1 and 2 have been considered, as explained in [15]. In addition, losses due to the finite conductivity of the metallic box are also taken into account. For the rectangular waveguide modes in media 1 and 3 (see Fig. 4), these losses are included using an attenuation term calculated, as in [21]. To consider the losses due to the metallic box walls in medium 2, the theory explained in [22] is used to compute the complex 
TABLE III

Physical Dimensions of the Transversal Filter, ACCORDING TO FIG. 4

\begin{tabular}{|c|c|}
\hline $\begin{array}{c}\text { Parameters } \\
\text { of the filter }\end{array}$ & $\begin{array}{c}\text { Dimensions } \\
\text { third filter } \\
\text { (transversal) }\end{array}$ \\
\hline \hline$h_{1}=h_{3}$ & $10 \mathrm{~mm}$ \\
$h_{2}$ & $2 \mathrm{~mm}$ \\
$l_{r 1}=l_{r 3}$ & $16.6 \mathrm{~mm}$ \\
$l_{r 2}$ & $30.2 \mathrm{~mm}$ \\
$w_{r 1}=w_{r 2}=w_{r 3}$ & $2 \mathrm{~mm}$ \\
$y_{r 1}=y_{r 3}$ & $16.8 \mathrm{~mm}$ \\
$y_{12}=y_{23}$ & $4.5 \mathrm{~mm}$ \\
$x_{12}=x_{23}$ & $11.1 \mathrm{~mm}$ \\
$a$ & $42.2 \mathrm{~mm}$ \\
$b$ & $48.6 \mathrm{~mm}$ \\
$\varepsilon_{r}$ & 2.1 \\
$Z_{p 1}=Z_{p 2}$ & $45 \Omega$ \\
\hline
\end{tabular}

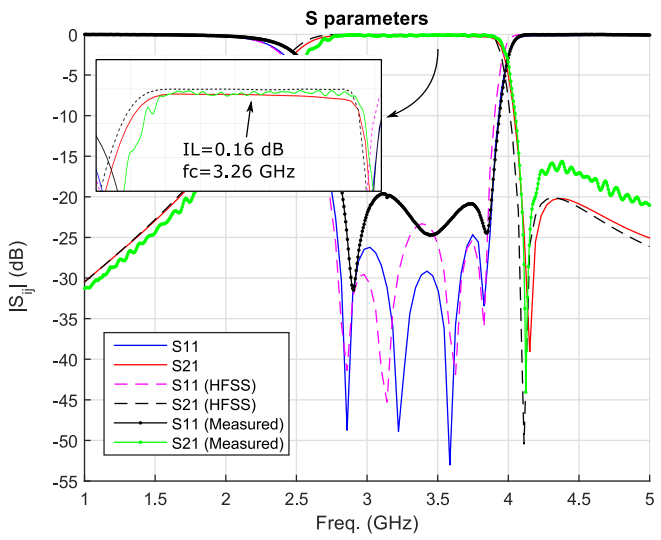

Fig. 11. Magnitude of the S-parameters for the transversal filter computed using the MEN formulation as compared with the HFSS results and to measurements from the prototype discussed in [19]. The thickness of the metallizations is $h_{2}=2 \mathrm{~mm}$. The MEN results are obtained with $\mathrm{dB}_{\mathrm{th}}=4 \mathrm{~dB}$, leading to $N=28$ accessible modes. We have used $\tan \delta=0.0037$ and $\sigma=3.8 \cdot 10^{7} \mathrm{~S} / \mathrm{m}$ to model the losses. Inset: Zoomed-in view of the $\left|S_{21}\right|$ parameter in the passband. In the zoomed-in view, the black curve is the HFSS result, the green curve is the measured response, and the red curve is the MEN simulation.

propagation constant for the arbitrary waveguide modes. This is an important difference with respect to the formulation presented in [15], where these losses are not considered because this waveguide was zero-thickness. Finally, the modes in media 1 and 3 of Fig. 3 are terminated with a surface impedance instead of a short circuit to model the losses in the top and bottom covers of the box enclosure (see [15] for more details). According to these considerations, we have used in the MEN results of Fig. 11, a loss tangent for the Teflon of $\tan \delta=0.0037$, and a conductivity for the box equal to $\sigma=3.8 \cdot 10^{7} \mathrm{~S} / \mathrm{m}$. A zoom of the insertion losses (IL) in the passband is shown in the inset of Fig. 11.

As can be seen, good agreement between simulations and measurements is also observed in this case. The IL in the MEN simulation agrees well with the IL reported in [19] ( $\mathrm{IL}=0.16 \mathrm{~dB}$ at the center frequency $f_{c}=3.26 \mathrm{GHz}$ ). The small differences between the simulations (MEN and HFSS) and the measured response could be due to manufacturing issues. For these simulations, we have selected $\mathrm{dB}_{\text {th }}=4 \mathrm{~dB}$, which leads to 28 accessible modes in the network of Fig. 3. In the MoM-Galerkin procedure, 200 basis and test functions have been used to expand the unknowns of the problem. Finally, 40 dynamic terms and 5000 static terms have been used in the kernel of the integral equations. The computational time required by the BI-RME code is 6 min. The MEN code for 100 frequency points takes only $20.1 \mathrm{~s}$. For completeness, HFSS takes more than 10 min to perform the same full-wave analysis. Note that for situations where the metallization does not change (for example, if we only modify the ports or the dielectric layers), then we would not need to recompute the BI-RME module and the total computational time would just be that corresponding to the MEN analysis (20.1 s).

In this example, we have also studied the effect of the thickness of the metallization on the numerical convergence of the algorithm. We have found that also for this structure, the condition on the power threshold can be relaxed when the thickness $h_{2}$ is reduced. Therefore, the number of accessible modes that need to be considered in the equivalent network does not increase substantially for thinner metallizations. In particular, the convergence study shows that if the bar resonators have a reduced thickness of $h_{2}=1 \mathrm{~mm}$, then the needed $\mathrm{dB}_{\text {th }}$ value is equal to $2 \mathrm{~dB}$, leading again to 28 accessible modes.

From this numerical example, we can conclude that the MEN technique for this kind of structure is both accurate and efficient. The results presented in Sections III and IV fully validate the MEN formulation applied to the analysis of microwave circuits composed of thick metallizations, including loss effects and the excitation through lateral coaxial connectors represented by transverse ports.

\section{CONClusion}

In this contribution, we have extended the MEN formulation to the analysis of shielded microwave devices consisting of several thick metallizations and dielectric layers, including the excitation through lateral ports representing coaxial connectors, internal ports, and loss effects. To rigorously account for the thick metallizations, the structure is decomposed into two discontinuities, which are characterized through their corresponding MENs. One of them contains the transverse ports that excite the structure. The two MENs are then cascaded to obtain the final network that models the complete structure. The numerical convergence of the technique with respect to the thickness of the metallizations has also been confirmed. Finally, several practical filters have been discussed, showing good agreement with respect to results from other commercial software tools and measurements, thereby fully validating the theoretical approach proposed in this contribution.

\section{REFERENCES}

[1] T. Itoh, Numerical Techniques for Microwave and Millimeter-Wave Passive Structures. Hoboken, NJ, USA: Wiley, 1989.

[2] J.-M. Jin, Theory and Computation of Electromagnetic Fields. Hoboken, NJ, USA: Wiley, 2015.

[3] M. Salazar Palma, T. K. Sarkar, L. E. Garcia Castillo, T. Roy, and A. Djordjevic, Iterative and Self-Adaptive Finite Elements in Electromagnetic Modeling, Norwood, MA, USA: Artech House, 1998.

[4] A. F. Peterson, S. L. Ray, and R. Mittra, Finite-Difference Time Domain Methods Orthogonal Meshes. Hoboken, NJ, USA: Wiley, 1998, pp. $495-523$.

[5] P. Arcioni, M. Bressan, and G. Conciauro, "Generalized admittance matrix of arbitrary E-plane waveguide junctions by the BI-RME method," in IEEE MTT-S Int. Microw. Symp. Dig., Denver, ON, USA, Jun. 199, pp. 211-214. 
[6] G. Conciauro, M. Guglielmi, and R. Sorrentino, Advanced Modal Analysis. CAD Techniques for Waveguide Components and Filters. New York, NY, USA: Wiley, 1999.

[7] H. K. Smith, J. R. Mosig, and M. Guglielmi, "Multimode network approach for the solution of two-dimensional waveguide discontinuities," in Proc. 23rd Eur. Microw. Conf., Sep. 1993, pp. 1-7.

[8] C. G. Molina, F. Q. Pereira, A. A. Melcon, V. B. Esbert, and M. Guglielmi, "Efficient formulation of multimode equivalent networks for 2-D waveguide steps through Kummer's transformation," in IEEE MTT-S Int. Microw. Symp. Dig., May 2017, pp. 1-9.

[9] C. Gómez Molina, F. Quesada Pereira, A. Álvarez Melcón, V. E. Boria, and M. Guglielmi, "An efficient technique to assess the convergence of the multimode equivalent network for waveguide devices," IEEE Trans. Microw. Theory Techn., vol. 66, no. 2, pp. 651-658, Feb. 2018.

[10] M. Guglielmi and A. A. Oliner, "Multimode network description of a planar periodic metal-strip grating at a dielectric interface. I. Rigorous network formulations," IEEE Trans. Microw. Theory Techn., vol. 37, no. 3, pp. 534-541, Mar. 1989.

[11] C. G. Molina et al., "Rigorous multimode equivalent network representation of multilayer planar circuits," in IEEE MTT-S Int. Microw. Symp. Dig., Aug. 2018, pp. 1-4.

[12] C. Gomez Molina, F. Quesada Pereira, A. Alvarez Melcon, V. E. Boria, and M. Guglielmi, "Electric multimode equivalent network technique for multilayer shielded circuits based on arbitrary rectangular elements," in Proc. Int. Conf. Electromagn. Adv. Appl. (ICEAA), Sep. 2019, pp. 1-5.

[13] T. C. Edwards and M. B. Steer, Foundations of Interconnect and Microstrip Design. New York, NY, USA: Wiley, 2000, doi: $10.1002 / 9781118894514$.

[14] M. A. Sanchez-Soriano et al., "Study on multipactor breakdown in coaxial to microstrip transitions," in IEEE MTT-S Int. Microw. Symp. Dig., Aug. 2018, pp. 1-7.

[15] C. G. Molina et al., "Multimode equivalent network for boxed multilayer arbitrary planar circuits," IEEE Trans. Microw. Theory Techn., early access, Mar. 15, 2020, doi: 10.1109/TMTT.2020.2984230.

[16] M. Bozzi, L. Perregrini, A. Alvarez Melcon, M. Guglielmi, and G. Conciauro, "MoM/BI-RME analysis of boxed MMICs with arbitrarily shaped metallizations," IEEE Trans. Microw. Theory Techn., vol. 49, no. 12 , pp. 2227-2234, Dec. 2001.

[17] S. Cogollos et al., "Efficient modal analysis of arbitrarily shaped waveguides composed of linear, circular, and elliptical arcs using the BI-RME method," IEEE Trans. Microw. Theory Techn., vol. 51, no. 12, pp. 2378-2390, Dec. 2003.

[18] I. Stevanovic, P. Crespo-Valero, and J. R. Mosig, "An integral-equation technique for solving thick irises in rectangular waveguides," IEEE Trans. Microw. Theory Techn., vol. 54, no. 1, pp. 189-197, Jan. 2006.

[19] C. Gomez-Molina et al., "Wideband bandpass filters using a novel thick metallization technology," IEEE Access, vol. 8, pp. 34962-34972, 2020, doi: 10.1109/ACCESS.2020.2974552.

[20] J. C. Rautio and R. F. Harrington, "An electromagnetic time-harmonic analysis of shielded microstrip circuits," IEEE Trans. Microw. Theory Techn., vol. 35, no. 8, pp. 726-730, Aug. 1987.

[21] N. Marcuvitz, Waveguide Handbook, MIT Radiation Laboratory Series, vol. 10. New York, NY, USA: Mc-Graw-Hill, 1951, pp. 57-60.

[22] S. Mariniet et al., "Rigorous evaluation of propagation losses in arbitrarily shaped waveguide structures using boundary integral-Resonant mode expansion and perturbation of boundary conditions," IET Microw., Antennas Propag., vol. 8, no. 12, pp. 980-989, Sep. 2014.

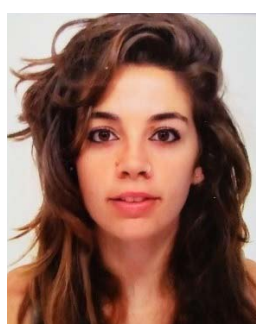

Celia Gomez Molina was born in Murcia, Spain, in 1992. She received the bachelor's degree in telecommunication systems and the master's degree in telecommunication engineering from the Technical University of Cartagena (UPCT), Murcia, in 2014 and 2016, respectively.

In 2016, she joined the Department of Information Technologies and Communications, UPCT, as a Researcher, where she is currently developing her teaching and Ph.D. research activities. She was a Visiting Student with the University of California at Davis, Davis, CA, USA, in 2018, and the Politecnico di Milano, Milan, Italy, in 2019. She is involved in the development of analytical and numerical tools for network representations of waveguide and planar discontinuities. Her current scientific interests include the design of microwave filters using different technologies.

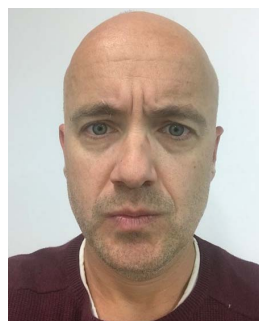

Fernando Quesada Pereira (Member, IEEE) was born in Murcia, Spain, in 1974. He received the master's degree in telecommunications engineering from the Technical University of Valencia (UPV), Valencia, Spain, in 2000, and the Ph.D. degree from the Technical University of Cartagena (UPCT), Murcia, in 2007.

In 1999, he joined the Department of Radiocommunications, UPV, as a Research Assistant, where he was involved in the development of numerical methods for the analysis of anechoic chambers and tag antennas. In 2001, he joined the Department of Communications and Information Technologies, UPCT, as a Research Assistant, and then as an Assistant Professor. In 2005, he was a Visiting Scientist with the University of Pavia, Pavia, Italy. In 2009, he was an Invited Researcher with UPV. In 2011, he became an Associate Professor with UPCT. His current research interest includes integral equation numerical methods for the analysis of antennas and microwave devices, along with microwave filters design and applications.

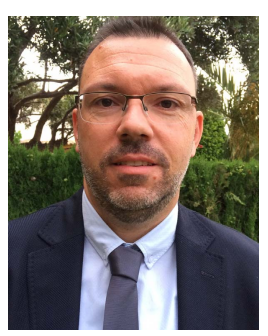

Stephan Marini (Senior Member, IEEE) received the Laurea degree in electronics engineering from the University of Perugia, Perugia, Italy, in 2001, and the Ph.D. degree in telecommunications from the Universidad Politécnica de Valencia, Valencia, Spain, in 2005

In 2001, he joined the Departamento de Comunicaciones, Universidad Politécnica de Valencia, where he was involved in the framework of the European Union Project-Millimeter-wave and Microwave Components Design Framework for Ground and Space Multimedia Network. Since 2005, he has been a Lecturer with the Departamento de Física, Ingeniería de Sistemas y Teoría de la Señal, Universidad de Alicante, Alicante, Spain, where he became an Associate Professor in 2012. In 2009, he became a fellow of the LEMA Laboratory, École Polytechnique Fédérale de Lausanne, Lausanne, Switzerland, as a Visiting Scientist, supported by the Spanish Government. His current research interests include numerical methods in computer-aided techniques for the analysis and design of microwave and millimeter passive components and simulation of power effects (multipactor and corona) in passive waveguide systems.

Dr. Marini currently serves as an Associate Editor for IET Electronics Letters, and a regular reviewer for the most relevant IEEE and IET technical journals in his areas of interest.

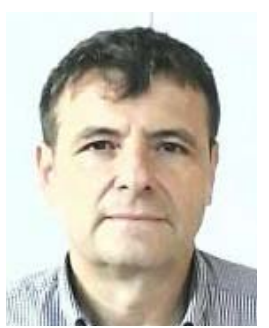

Alejandro Alvarez Melcon (Senior Member, IEEE) was born in Madrid, Spain, in 1965. He received the master's degree in telecommunications engineering from the Technical University of Madrid (UPM), Madrid, in 1991, and the Ph.D. degree in electrical engineering from the ETH Zürich, Lausanne, Switzerland, in 1998

In 1988, he joined the Department of Signal, Systems and Radiocommunications, UPM, as a Researcher, where he was involved in the design, testing, and measurement of broadband spiral antennas for electromagnetic measurements support equipment. From 1991 to 1993, he was with the Radio Frequency Systems Division, European Space Agency (ESA/ESTEC), Noordwijk, The Netherlands, where he was involved in the development of analytical and numerical tools for the study of waveguide discontinuities, planar transmission lines, and microwave filters. From 1993 to 1995, he was with the Space Division, Industry Alcatel Espacio, Madrid, and also with the ESA, where he collaborated in several ESA/European Space Research and Technology Centre contracts. From 1995 to 1999, he was with the ETH Zürich, Ecole Polytechnique Fédérale de Lausanne, Lausanne, where he was involved with the field of microstrip antennas and printed circuits for space applications. In 2000, he joined the Technical University of Cartagena, Murcia, Spain, where he is currently developing his teaching and research activities. He was an Invited Professor with the Polytechnique Montréal, Montreal, QC, Canada, in 2010, and a Visiting Professor with the University of California at Davis, Davis, CA, USA, from 2017 to 2018.

Dr. Alvarez Melcón was a recipient of the Journée Internationales de Nice Sur les Antennes (JINA) Best Paper Award for the best contribution to the JINA'98 International Symposium on Antennas and the Colegio Oficial de Ingenieros de Telecomunicación Award to the best Ph.D. dissertation in basic information and communication technologies. 
This article has been accepted for inclusion in a future issue of this journal. Content is final as presented, with the exception of pagination.

10

IEEE TRANSACTIONS ON MICROWAVE THEORY AND TECHNIQUES

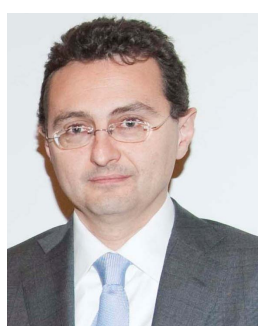

Vicente E. Boria (Fellow, IEEE) was born in Valencia, Spain, in 1970. He received the master's degree (Hons.) in telecommunications engineering and the Ph.D. degree in telecommunications engineering from the Universidad Politécnica de Valencia, Valencia, in 1993 and 1997, respectively.

In 1993, he joined the Departamento de Comunicacrones, Universidad Politécnica de Valencia, where he has been a Full Professor since 2003. In 1995 and 1996, he was holding a Spanish trainee position with the European Space Research and Technolorgy Centre, European Space Agency, Noordwijk, The Netherlands, where he was involved in the area of electromagnetic analysis and design of passive waveguide devices. He has authored or coauthored 15 chapters in technical textbooks, 180 articles in refereed international technical journals, and over 200 articles in international conference proceedings. His current research interests are focused on the analysis and automated design of passive components, particularly in filters and multiplexers, several technologies, and the simulation and measurement of power effects in high-frequency devices and systems.

Dr. Beria has been a member of the IEEE Microwave Theory and Techniques Society (IEEE MTT) and the IEEE Antennas and Propagation Society since 1992. He is currently a member of the European Microwave Association (EuMA). He is also an Editorial Board Member of the International Journal of RF and Microwave Computer-Aided Engineering and a member of the Technical Committees of the IEEE-MTT International Microwave Symposium and the European Microwave Conference. He has been the Chair of the 48th European Microwave Conference held in Madrid, Spain. He acts as a regular reviewer of the most relevant IEEE and IET technical journals on his areas of interest. He was an Associate Editor of the IEEE MICROWAVE AND WIRELESS COMPONENTS LETTERS from 2013 to 2018 and the IET Electronics Letters from 2015 to 2018 . He currently serves as a Subject Editor of the IET Electronics Letters (Microwaves).

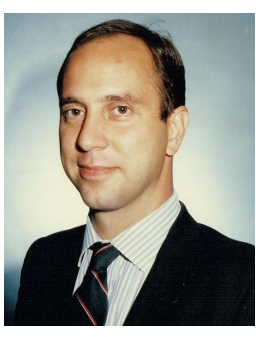

Marco Guglielmi (Life Fellow, IEEE) was born in Rome, Italy, in 1954. He received the Laura degree in electronic engineering from the Sapienza University of Rome, Rome, Italy, in 1979, the M.S. degree in electrical engineering from the University of Bridgeport, Bridgeport, CT, USA, in 1982, and the Ph.D. degree in electrophysics from the Polytechnic University, Brooklyn, NY, USA, in 1986.

In 1980, he also attended the Scuola di Opecializzazione in Elettromagnetismo Applicator at the Sapienza University of Rome. From 1984 to 1986 , he was an Academic Associate with Polytechnic University, Brooklyn, NY, USA, where he was an Assistant Professor from 1986 to 1988. From 1988 to 1989 , he was an Assistant Professor with the New Jersey Institute of Technology, Newark, NJ, USA. In 1989, he joined the European Space Agency as a Senior Microwave Engineer with the RF System Division, European Space Research and Technology Centre (ESTEC), Noordwijk, The Netherlands, where he was in charge of the development of microwave filters and electromagnetic simulation tools. In 2001, he was appointed the Head of the Technology Strategy Section, ESTEC, where he contributed to the development of management processes and tools for the formulation of a European strategy for space technology research and development. In 2014, he retired from the European Space Agency. He is currently holding an invited senior researcher position with the Polytechnic University of Valencia, Valencia, Spain. In 1981, he received a Fulbright Scholarship in Rome and a Halsey International Scholarship Program for his M.S. studies.

Dr. Guglielmi has been elevated to the grade of fellow of the IEEE in 2013 for contributions to multimode equivalent networks and microwave filter design.

Authorized licensed use limited to: Stephan Marine. Downloaded on August 29,2020 at 11:34:51 UTC from IEEE Xplore. Restrictions apply. 